\title{
Privacy Preservation by $k$-Anonymization of Weighted Social Networks
}

\author{
Maria E. Skarkala, Manolis Maragoudakis, \\ Stefanos Gritzalis and Lilian Mitrou \\ Department of Information and Communication \\ Systems Engineering \\ University of the Aegean \\ Karlovassi, Samos 83200, Greece \\ Email: \{mes, mmarag, sgritz, l.mitrou\}@aegean.gr
}

\author{
Hannu Toivonen and Pirjo Moen \\ Department of Computer Science and HIIT \\ University of Helsinki, Finland \\ Email: \{hannu.toivonen, pirjo.moen\}@cs.helsinki.fi
}

\begin{abstract}
Privacy preserving analysis of a social network aims at a better understanding of the network and its behavior, while at the same time protecting the privacy of its individuals. We propose an anonymization method for weighted graphs, i.e., for social networks where the strengths of links are important. This is in contrast with many previous studies which only consider unweighted graphs. Weights can be essential for social network analysis, but they pose new challenges to privacy preserving network analysis. In this paper, we mainly consider prevention of identity disclosure, but we also touch on edge and edge weight disclosure in weighted graphs. We propose a method that provides $k$-anonymity of nodes against attacks where the adversary has information about the structure of the network, including its edge weights. The method is efficient, and it has been evaluated in terms of privacy and utility on real word datasets.
\end{abstract}

\section{INTRODUCTION}

Social networks can be analyzed and mined for various interesting questions, such as how opinions spread or how communities evolve. As social networks are released, there is an increasing concern about the privacy of individuals involved [12], [18]. Therefore, before publishing networks, it is necessary to ensure that they do not disclose sensitive information, such as identities of the individuals or their relationships [20]. The problem is not trivial, since simply removing all identifiers or replacing them with anonymous ones is insufficient [1], [5].

Potential privacy breaches in social networks can be categorized into three types: identity disclosure, link disclosure, and content disclosure [3]. Individual identity is disclosed when an adversary is able to identify a target individual in the network. Link disclosure occurs when the existence of a relationship between two given individuals can be uncovered. Content disclosure, in turn, means that data associated with a node (individual) or an edge (relationship between two individuals) are made available.

In many social networks, edge weights have a special role since social ties between individuals may be stronger or weaker [8]. However, edge weights pose challenges for network anonymization. On one hand, an adversary who has information about the edge weights of individuals can use that information to attack the network. At the same time, since edge weights are crucial for many analyses of weighted graphs, as much information should be maintained as possible, to preserve the utility of the anonymized data [17].

We propose a novel $k$-anonymization [14] method of weighted social network data, to prevent identity disclosure by making each individual indistinguishable from at least $k-1$ other individuals. In networks, these techniques can be categorized into two general categories: (1) clustering or generalization-based and (2) graph modification-based ones. Our approach falls in the first category: it groups similar nodes (and edges) together and generalizes them to be identical and, thus, indistinguishable within each group. The proposed method is inspired by work on network compression [15]. The second approach, in turn, modifies the topological structure of the graph to make nodes structurally identical. The generalization-based approach we use gives a stronger protection against identity disclosure, as it includes degree anonymity, weight bag anonymity, as well as neighborhood anonymity as its special cases.

In addition to preventing identity disclosure in weighted networks, our method can optionally prevent edge disclosure and edge weight disclosure, if needed. For both of them, the $k$-anonymization process usually introduces some uncertainty. The amount of this uncertainty in an anonymized graph can be quantified, and also increased if needed to achieve the required privacy level.

The rest of the paper is structured as follows. The problem and key concepts are defined in Section II. We propose a novel algorithm for weighted graph anonymization in Section III. In Section IV we evaluate the algorithm experimentally using real data. Section V reviews previous work on privacy preservation over graphs and networks. Finally, a brief conclusion is given in Section VI.

\section{Problem Definition}

We model social networks as graphs that are weighted and undirected. A weighted graph is defined as a triple $G=$ $(V, E, W)$ where $V$ is a set of nodes, $E$ a set of edges, and 
$W$ a positive weight function. Given an edge $e_{i, j}=(i, j) \in$ $E \subset V \times V$, its weight is denoted by $w_{i, j}=W(i, j)$.

We consider the following abstract problem. Given a weighted graph $G$, produce a privacy-preserving version $G^{\prime}$ of it.

Preventing node identity disclosure: The method we consider provides $k$-anonymity [14] of individuals in the network.

Definition 1: A graph is $k$-anonymous if every node in it is indistinguishable from at least $k-1$ other nodes.

In our method the basic idea is that original nodes in graph $G$ are grouped into supernodes, and edges between the original nodes are replaced by superedges between the supernodes. A supernode represents all the original nodes it contains, and a relationship between any two original nodes is described by a superedge between supernodes.

Definition 2: A supernode $s n_{k}$ in an anonymized graph $G^{\prime}$ represents a set of original nodes $n_{i}$ in graph $G$.

Definition 3: A superedge $e_{s n_{i}, s n_{j}}$ in an anonymized graph $G^{\prime}$ represents all possible edges between nodes in supernodes $s n_{i}$ and $s n_{j}$.

While a supernode is simply a group of nodes, a superedge represents a hypothetical set of edges. This set may contain edges that do not exist in the original graph $G$. These give rise to information loss, which we will discuss shortly.

To produce a $k$-anonymous generalised graph, we group the original nodes of $G$ into supernodes of size at least $k$.

Definition 4: A $k$-anonymity grouping of a graph $G=$ $(V, E, W)$ is a partitioning of the nodes in set $V$ into supernodes $s n_{i}$ such that $\left|s n_{i}\right| \geq k$.

A graph $G^{\prime}=\left(V^{\prime}, E^{\prime}, W^{\prime}\right)$ is $k$-anonymous if the set $V^{\prime}$ of supernodes is a $k$-anonymity grouping of the set $V$ of original nodes. This follows from the fact that a supernode represents all of its original nodes, so that the at least $k$ original nodes within a supernode become undistinguishable.

A $k$-anonymous graph $G^{\prime}$ now consists of supernodes and superedges. For analysis, the process of grouping nodes and edges has to reversed to recover an approximate copy of the original graph. Original nodes are easily recovered from supernodes, but edges and their weights may have changed (we will return to this shortly). In particular, a superedge is associated with the count of true edges it represents but not their identities. To obtain an approximation of the original graph, that count of edges is materialized among all the possible edges a superedge represents. Since this results in some random changes of the network topology, it is a good practice to produce a number of alternative reconstructions, analyze all of them, and study the statistics over these graphs.

The problem can now be stated more exactly as follows.

Problem: Given a weighted graph $G=(V, E, W)$, find a $k$-anonymous graph $G^{\prime}=\left(V^{\prime}, E^{\prime}, W^{\prime}\right)$ such that a given information loss measure $I L\left(G, G^{\prime}\right)$ is minimized.
Preventing edge disclosure: Information on relationships between individuals may also be considered sensitive. In such cases, the anonymization of the network data should also protect the information on connections between individuals, i.e., to prevent edge and edge weight disclosure. However, the $k$-anonymity as described above is not necessarily sufficient to prevent these two types of disclosure [11].

One possibility to prevent or at least make it more difficult for an adversary to get definite information on the existence of connections between nodes, is to avoid superedges that give absolute information about the existence of original edges. Due to $k$-anonymity grouping, a superedge typically also represents edges that do not exist in the original graph, and therefore, this can be done easily using superedge probabilities.

Definition 5: A superedge probability $p_{s n_{i}, s n_{j}}$ describes how certain (or uncertain) the existence of an edge between any pair of nodes included in supernodes $s n_{i}$ and $s n_{j}$ is.

Such a superedge probability can be defined as the percentage of original edges that are represented by a superedge. By keeping these probabilities below a given threshold $p^{\prime}$, an adversary can only infer the existence of an edge at most with confidence $p^{\prime}$, assuming that the adversary does not have any other relevant information about the original network.

Preventing edge weight disclosure: Edge weights give descriptive information about the relationships between two nodes in a graph. Such information can be seen as sensitive, and if an adversary is aware of such weights, this information can be used to identify connected target nodes even in an anonymized graph. Thus, protection of the edge weights, i.e., avoiding edge weight disclosure, should also be ensured before publishing the network data.

In the case of $k$-anonymity grouping, the weights assigned to the superedges are combined from the original edge weights $w_{i, j}$, since the edges $e_{i, j}$ between the original nodes are joined to the superedges. The weights of these superedges are called superedge weights $w_{s n_{i}, s n_{j}}$. The combination of the original edge weights can be done in different ways. In our approach, a superedge weight is defined as the average of the original edge weights, i.e., the superedge weight is

$W^{\prime}\left(s n_{i}, s n_{j}\right)=\frac{\sum_{e \in I J} w_{e}}{|I J|}$, where $I J=E \cap\left(s n_{i} \times s n_{j}\right)$.

Often, these superedge weights $w_{s n_{i}, s n_{j}}$ differ from the weights $w_{i, j}$ of the original edges. Thus, exact edge weight disclosure in those cases is prevented if the adversary has no other information. If the adversary knows all but one weight, that weight can be reverse-engineered. For more systematic and controlled protection, superedge weights can be modified by a random component to add uncertainty of their real values. 
Measuring information loss: So far we have not considered at all how much information is changed or lost in the process of network anonymization. Ultimately, the amount of information lost depends on the application. Internally, the anonymization algorithm also uses an information loss function: the goal is to minimize information loss under the constraint that $k$-anonymity has to be reached. This loss function serves as a proxy of the real and unknown information loss functions of applications.

In our algorithm, we use as information loss function the edge weight dissimilarity between the original graph $G$ and the generalised graph $G^{\prime}$ :

$$
I L\left(G, G^{\prime}\right)=\sum_{(i, j) \in E}\left|W(i, j)-W^{\prime}(i, j)\right|^{2},
$$

where $W^{\prime}(i, j)$ is the weight of the superedge that represents edge $(i, j)$. It can be shown that the sum in Equation 2 is minimized when the weight of each superedge is the average of the original edge weights as was defined in Equation 1.

\section{Algorithm Description}

Based on the $k$-anonymity model, the main goals of our proposal are to prevent identity, edge and edge weight disclosure by an adversary which possesses knowledge about the structure of the original graph. The basic steps of our proposal for social network anonymization are the following:

Step 1 (Naive Anonymization). All the identifiers of the original graph $G$ are removed and replaced by temporary identities. By applying only this step, an adversary who does not possess any prior knowledge on $G$ cannot re-identify any targeted node, edge or edge weight.

Step 2 (Node anonymity). In order to obtain a $k$ anonymity grouping of graph $G$, the original nodes of the graph are grouped to supernodes based on the similarity and strength of their relationships to other nodes. $k$-anonymity grouping indicates that each supernode should include at least $k$ original nodes. The only immediate information about the supernodes is their supernode membership.

Step 3 (Edge weight computation). The original edges that connect nodes in graph $G$ are grouped and represented by superedges between supernodes in graph $G^{\prime}$. The weights assigned to the superedges are combined from the original edge weights $w_{i, j}$, and result from Equation 1, with the possible addition of a random component for further protection of edge weights.

Step 4 (Edge anonymity). In addition to the edge weight $w^{\prime}$, the superedges are also described by the probability of edge existence $p_{s n_{i}, s n_{j}}$, which defines the percentage of original edges that are represented by a superedge. If this probability is higher than $p$ (a user given parameter) then the probability is bounded by a threshold $p^{\prime}$.

Step 5 (Publication of the anonymized network). The final anonymized network $G^{\prime}$ is released for analysis.

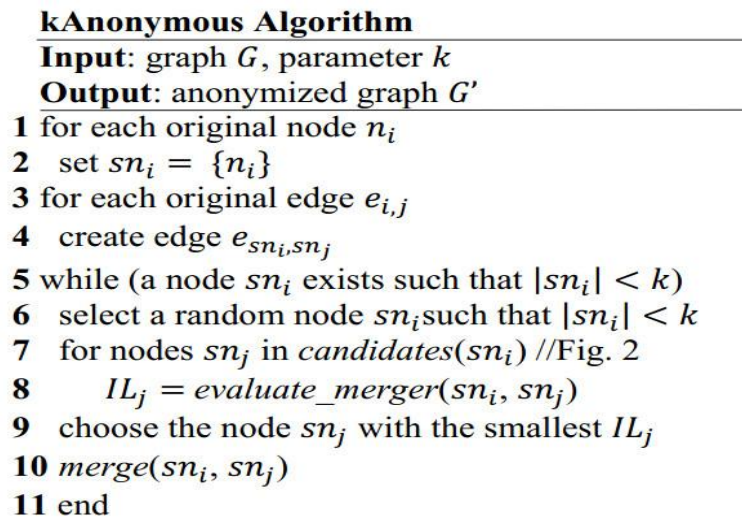

Figure 1: kAnonymous Algorithm

The proposed method is described in kAnonymous algorithm (Figure 1), which takes as input a weighted graph $G$ and a parameter $k$ and returns an anonymized graph $G^{\prime}$. First, each original node $n_{i}$ in graph $G$ is described as a supernode $s n_{i}$ in graph $G^{\prime}$ and the corresponding edges between the supernodes are created $\left(e_{s n_{i}, s n_{j}}=e_{i, j}\right)$. Line 5 examines if there exists a supernode $s n_{i}$ which contains less than $k$ nodes, i.e. if the network stills needs to be anonymized. If yes, then on Line 6 such a supernode is picked at random. On Lines 7-10 it is merged with another supernode. To implement different strategies for selecting this other supernode, a separate function candidates returns a list of possible options (see below). Each of these candidates is considered for possible merger in turn (Line 8), and finally the best candidate is chosen.

The candidates function (Figure 2) returns a set of candidate nodes with which node $s n_{i}$ could be merged. Since nodes with shared neighbors are likely to merge best, the set of 2-hop neighbors of node $s n_{i}$ is used as candidates. In the rare occasion that this set is empty, the set of neighbors constitutes the next attempted candidate set. If this set is empty, too, then all remaining supernodes are used as candidates. The merger_candidates function further constraints the candidate set. It returns either (1) a single random supernode from the candidate set, (2) the whole set of candidates, or (3) only those candidates that are themselves not yet $k$-anonymous. In Section IV, we evaluate these three versions of our proposal since they give different trade-offs between speed and utility. The evaluate_merger function computes the information loss, using Equation 2, for grouping supernode $s n_{i}$ with a possible candidate $s n_{j}$. Function merge creates a new supernode $s n_{n e w}$ as the merger of supernode $s n_{i}$ with supernode $s n_{j}$, and it creates all superedges related to this new supernode and assigns them the average weight $w^{\prime}$ of the corresponding edges. The $k$ Anonymous algorithm iterates until all the supernodes in graph $G^{\prime}$ represent at least $k$ nodes of graph $G$. 


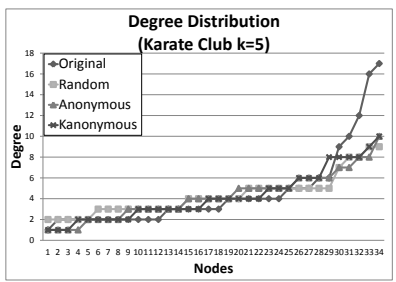

Nodes
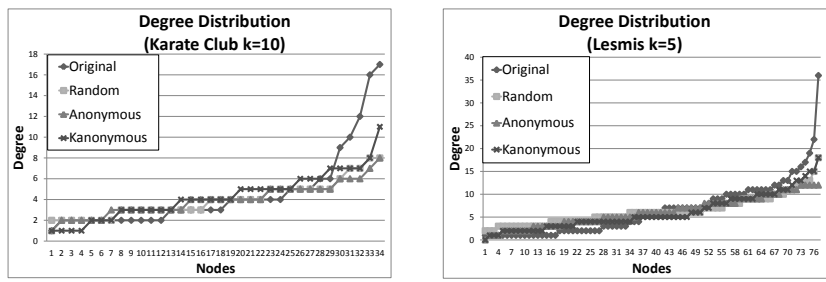

Figure 3: Degree Distribution
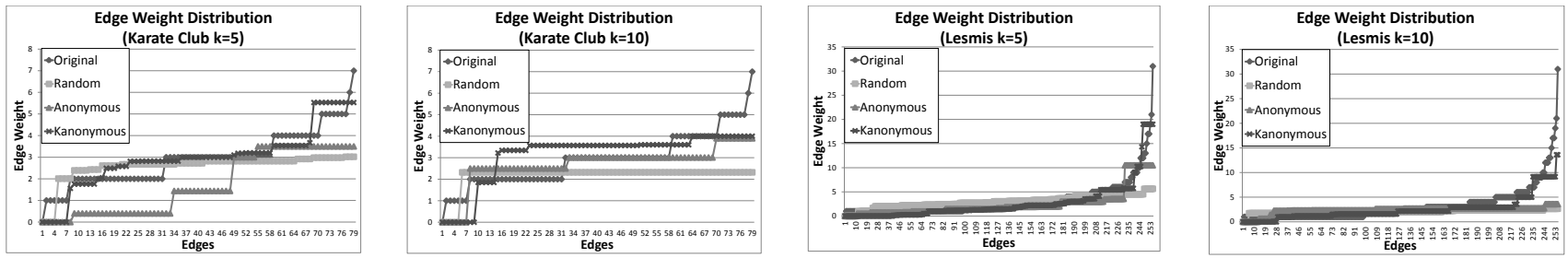

Figure 4: Edge Weight Distribution
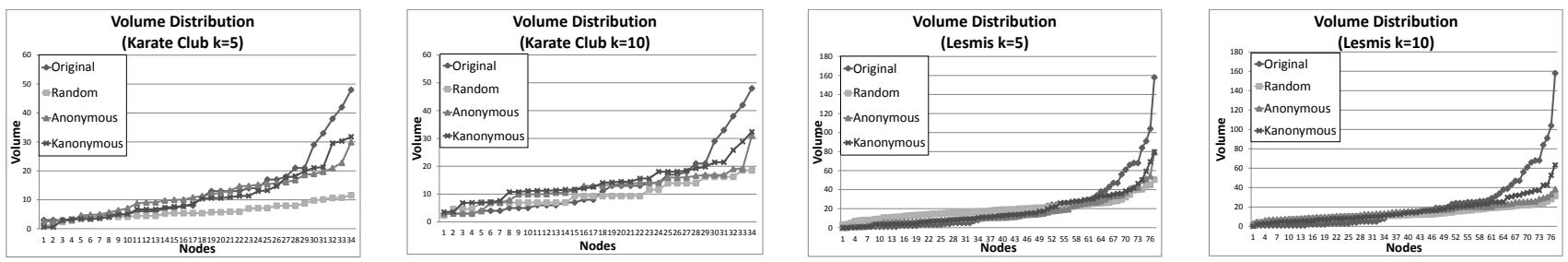

Figure 5: Volume Distribution
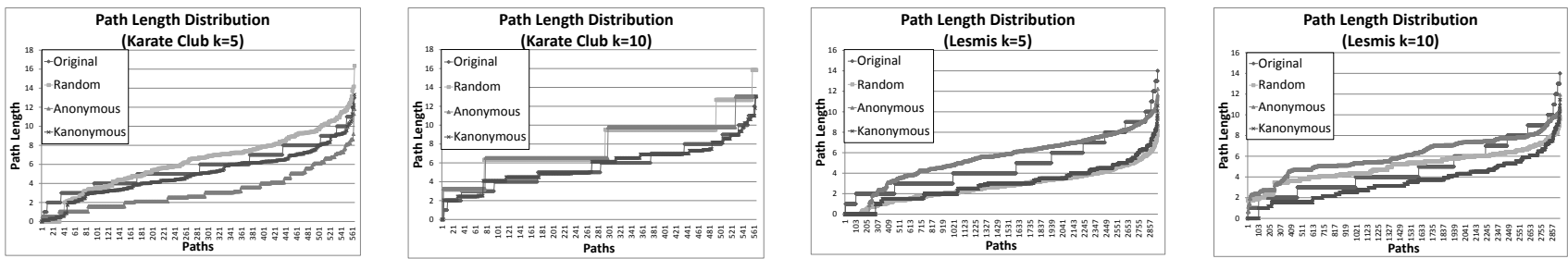

Figure 6: Path Length Distribution

Most of the existing literature on network anonymization deals with privacy preservation on simple undirected and unweighted graphs. There are, however, some methods for weighted networks.

Liu et al. (e.g., [13]) have studied anonymization techniques for weighted graphs to preserve linear properties such as shortest paths between pairs of nodes. Das et al. [4] propose a linear programming method to anonymize the weights of edges while preserving shortest paths. Li et al. [8] propose volume and histogram anonymization to prevent weight-based attacks by modifying edges and edge weights.
They also propose a volume sequence perturbation model for weight anonymization [9]. A greedy algorithm is proposed by Wang et al. [16] to preserve sensitive paths. The method perturbs a minimal number of edge weights so that there are at least $k$ indistinguishable shortest paths.

A generalization-based approach that preserves a network from identity and edge weight disclosure, is presented by Liu et al. [10]. Their method groups together nodes with similar weight bags, i.e., with a similar set of weights, not with a similar set of neighbors. For a fixed $k$, our model gives less information to the adversary, but may also have lower utility. 
Comparing these approaches is a topic for future work.

\section{CONCLUSION}

Publishing social network data for analysis by researchers while preserving the privacy of the individuals involved has raised many concerns. In this paper we presented a clustering-based $k$-anonymization technique for weighted network data. The method groups nodes with similar sets of neighbors and their connections into supernodes and superedges, respectively. At the same time, the method tries to preserve utility of the graph. Experimental results suggest that the approach can find a balance between privacy and utility for real world weighted graphs. Further research work can be carried out to investigate the performance of our proposal in more complex social networks, its effectiveness on other statistical graph properties, and to compare it to other approaches.

\section{ACKNOWLEDGEMENTS}

This work has been supported by the Algorithmic Data Analysis (Algodan) Center of Excellence of the Academy of Finland.

\section{REFERENCES}

[1] Backstrom L., Dwork C., and Kleinberg J. "Wherefore art thou r3579x?: anonymized social networks, hidden patterns, and structural steganography". In Proceedings of the 16th international conference on World Wide Web (WWW '07). ACM, New York, NY, USA, pp. 181-190, 2007.

[2] Campan A. and Truta T.M. "A clustering approach for data and structural anonymity in social networks". In Proceedings of the 2nd ACM SIGKDD International Workshop on Privacy, Security, and Trust in KDD (PinKDD'08), in Conjunction with KDD'08, Las Vegas, Nevada, USA, pp. 1-10, 2008.

[3] Clarkson K., Liu K., and Terzi E. "Towards identity anonymization in social networks". In Link Mining: Models Algorithms and Applications, Chapter 14, pp. 359385 , Springer, 2010.

[4] Das, S., Omer E. and Abbadi, A. E. "Anonymizing edgeweighted social network graphs". Technical report, Department of Computer Science, University of California Santa Barbara, USA, 2009.

[5] Hay M., Miklau G., Jensen D., Weis P., and Srivastava S. "Anonymizing social networks". Technical Report 07-19, University of Massachusetts Amherst, 2007.

[6] Hay M., Miklau G., Jensen D., Towsley D., and Weis P. "Resisting structural re-identification in anonymized social networks". In Proc. VLDB Endow. vol. 1, no. 1, pp. 102$114,2008$.

[7] Knuth D.E. "The Stanford GraphBase: A platform for Combinatorial Computing", Addison-Wesley, Reading, MA, 1993.
[8] Li Y. and Shen H. "Anonymizing Graphs Against WeightBased Attacks". In Proceedings of the 2010 IEEE International Conference on Data Mining Workshops (ICDMW '10). IEEE Computer Society, Washington, DC, USA, pp. 491-498, 2010.

[9] Li Y. and Shen H. "On Identity Disclosure in Weighted Graphs". In Proceedings of the 2010 International Conference on Parallel and Distributed Computing, Applications and Technologies (PDCAT '10). IEEE Computer Society, Washington, DC, USA, pp. 166-174, 2010.

[10] Liu X. and Yang X. ”A generalization based approach for anonymizing weighted social network graphs". In Proceedings of the 12th international conference on Web-age information management (WAIM'11), Springer-Verlag Berlin, Heidelberg, pp. 118-130, 2011.

[11] Liu, L., Liu, J., and Zhang, J. "Privacy preservation of affinities in social networks". In Proceedings of the International conference on Information Systems, pp. 372-376, Porto, Portugal, 2010.

[12] Liu K., Das K., Grandison T., and Kargupta H. "PrivacyPreserving Data Analysis on Graphs and Social Networks". In Next Generation of Data Mining, Chapter 21, pp. 419-437, Chapman \& Hall/CRC, December 2008.

[13] Liu, L., Wang, J., Liu, J., and Zhang, J. "Privacy preservation in social networks with sensitive edge weights". In SIAM Data Mining Conference (SDM 2009), pp. 954965, 2009.

[14] Samarati P., "Protecting Respondent's Privacy in Microdata Release", IEEE Transactions on Knowledge and Data Engineering, vol. 13, n. 6, pp. 1010-1027, November 2001.

[15] Toivonen H., Zhou F., Hartikainen A., and Hinkka A. "Compression of weighted graphs". In Proceedings of the 17th ACM SIGKDD international conference on Knowledge discovery and data mining (KDD '11). ACM, New York, NY, USA, pp. 965-973, 2011.

[16] Wang S.L., Tsai Z.Z., Hong T.P., and Ting I.H. "Anonymizing shortest paths on social network graphs". In Proceedings of the Third international conference on Intelligent information and database systems - Volume Part I (ACIIDS'11), SpringerVerlag Berlin, Heidelberg, pp. 129-136, 2011.

[17] Watanabe C., Amasaga T. and Liu L.. "Privacy Risks and Countermeasures in Publishing and Mining Social Network Data". In Proceedings of International Conference on Collaborative Computing (CollaborateCom 2011), Orlando, FL.USA, pp. 55-66, Oct. 2011.

[18] Wu X., Ying X., Liu K., and Chen L. ”A survey of algorithms for privacy- preservation of graphs and social networks" In Managing and Mining Graph Data. Chapter 14, Kluwer Academic Publishers, vol. 40, pp. 421-453, 2010

[19] Zachary W. "An information flow model for conflict and fission in small groups". Journal of Anthropological Research, vol. 33, no. 4, pp. 452-473, 1977.

[20] Zou L., Chen L., and Ozsu M.T. ”k-automorphism: a general framework for privacy preserving network publication". Proc. VLDB Endow. 2, 1, pp. 946-957, 2009. 